\title{
ON A REVISED ANALYTIC NETWORK PROCESS (ANP) FOR AVOIDING IRRATIONAL RANK REVERSAL - A Revised Normalization Procedure and Calculation Method -
}

\author{
Kouichi Taji, Yousuke Sagayama and Hiroyuki Tamura \\ Graduate School of Engineering Science, Osaka university \\ 1-3 Machikaneyama, Toyonaka, Osaka 560-8531, JAPAN \\ \{taji, tamura\}@sys.es.osaka-u.ac.jp, saga@tamlab.sys.es.osaka-u.ac.jp
}

\begin{abstract}
In this paper we propose a revised ANP by using the aspiration level of decision maker. In the ordinary ANP, the irrational rank reversal of alternatives may arise when we add another alternative or extract an alternative. Such irrational rank reversal is caused by the ordinary normalization procedure in which the weights of alternatives sum to 1. To avoid this, we introduce the revised normalization procedure in which the aspiration level determined by the decision maker for each criterion equals to 1 . But since, by using this normalization, the resulting supermatrix may not be a stochastic matrix, the weight vector doesn't converge to a constant vector when the power method is used. So, we also propose the calculation method for relative importance based on the Perron-Frobenius theorem. A simple example shows that the irrational rank reversal is avoided in the proposed ANP.
\end{abstract}

\section{Introduction}

ANP (Saaty, 1996) is one of decision making models which includes the AHP (Saaty, 1980) as a special case. Hence, the ANP inherits the defects of AHP. For example, the irrational rank reversal phenomenon often seen in AHP (Belton and Gear, 1983 and 1985) also occurs in ANP. For example, even if we add a new alternative which is a copy of the one of current alternatives, the irrational rank reversal can occur. It is known that the irrational rank reversal can occur because of the ordinary normalization procedure, in which the weights of alternatives sum to 1. But in ANP, there exist other rank reversal which are not due to the normalization procedure. This rank reversal occurs because the weights of criteria change when adding another alternative or extracting an alternative. This implies that such rank reversal is not irrational and we accept such rational rank reversal which arises due to the structure of ANP.

In the case of AHP, it is shown (Tamura et al., 1998) that the irrational rank reversal can be avoided by using the revised normalization procedure. Their normalization method exploits the aspiration level of the decision maker and normalizes the weights of each alternatives so that the aspiration level equals to 1 .

In this paper, we propose a revised ANP to remove the irrational rank reversal. The proposed ANP also exploits the aspiration level of the decision maker and uses the revised normalization procedure. However, the revised normalization causes another problem such that the supermatrix may not be a stochastic matrix, and hence the relative importance of alternatives can not be computed by the power method used in ordinary ANP. Therefore, we also propose a modified power method based on the Perron-Frobenius theorem (Bertsekas and Tsitsiklis, 1989) to compute the relative importance of alternatives. A small example shows that the irrational rank reversal owing to the normalization procedure is avoided in the revised ANP. 


\section{The Irrational Rank Reversal Owing to Ordinary Normalization}

In this section, we explain by a simple example of AHP why the ordinary normalization procedure causes the irrational rank reversal.

Let consider the example consisting of two alternatives, $a_{1}$ and $a_{2}$, and two criteria, $c_{1}$ and $c_{2}$. By performing pairwise comparison for criteria, we have the weights of $c_{1}$ and $c_{2}$ as 0.667 and 0.333 , respectively. Also as shown in Table 1 , the weights of $a_{1}$ and $a_{2}$ under each criterion are obtained through pairwise comparison and the ordinary normalization in which the weights sum to 1 . Then the relative importance are computed as $a_{1}=0.511$ and $a_{2}=0.489$, that is, $a_{1}$ is more important than $a_{2}$.

Table 1 : Weights and relative importance of two alternatives

\begin{tabular}{|c|c|c|c|}
\hline & weight under $c_{1}$ & weight under $c_{2}$ & relative importance \\
\hline$a_{1}$ & 0.667 & 0.200 & 0.511 \\
\hline$a_{2}$ & 0.333 & 0.800 & 0.489 \\
\hline
\end{tabular}

Now, suppose that a new alternative $a_{3}$ is added. By performing the pairwise comparison again, the normalized weights of alternatives are computed as shown in Table 2 . From these weights the relative importance of alternatives are computed as $a_{1}=0.204, a_{2}=0.296$ and $a_{3}=0.500$, and hence, the rank reversal between $a_{1}$ and $a_{2}$ occur.

Table 2 : Weights and relative importance of three alternatives

\begin{tabular}{|c|c|c|c|}
\hline & weight under $c_{1}$ & weight under $c_{2}$ & relative importance \\
\hline$a_{1}$ & 0.222 & 0.167 & 0.204 \\
\hline$a_{2}$ & 0.111 & 0.666 & 0.296 \\
\hline$a_{3}$ & 0.667 & 0.167 & 0.500 \\
\hline
\end{tabular}

We think that this rank reversal is irrational because the alternative $a_{3}$ essentially must be evaluated independently on $a_{1}$ and $a_{2}$, that is, $a_{3}$ must not affect on the relative importance only between $a_{1}$ and $a_{2}$. Table 1 and 2 show that, when $a_{3}$ is added, the ratio of weight of $a_{1}$ to that of $a_{2}$ under each criterion does not change. On the other hand, the ratio of the weight of $a_{i}(i=1,2)$ under $c_{1}$ to that under $c_{2}$ changes significantly. This is caused by the fact that the weights of $a_{3}$ under $c_{1}$ and $c_{2}$ are much different and that the weights are normalized as the weights of alternatives sum to 1 . In this way, the irrational rank reversal owing to the ordinary normalization occurs.

\section{Revised ANP with Aspiration Level}

To avoid the irrational rank reversal, we propose the revised ANP by introducing the aspiration level. The aspiration level is the lowest level regarded by the decision maker. For example, in the case of car purchase, the aspiration level for the price may correspond to the budget of the decision maker. The aspiration level for the qualitative criterion may be the alternative which is the nearest to the ideal of decision maker. 
We first explain the revised normalization procedure using the aspiration level. In the revised normalization, the decision maker performs pairwise comparison under each criterion among alternatives consisting of actual alternatives and the imaginary alternative which has the aspiration level of decision maker. Then the weights of alternatives are normalized in a way that the aspiration level equals to 1 . It had been shown (Tamura et al., 1998) that the irrational rank reversal can be avoided by using the revised procedure in AHP. So we introduce this revised normalization procedure to ANP.

However, the revised normalization procedure causes another issue such that the resulting supermatrix may not be a stochastic matrix. Hence, the relative importance of alternatives can not be computed when the ordinary power method is used. Therefore, we propose the revised power method based on the PerronFrobenius theorem to compute the relative importance of alternatives. Let consider the supermatrix $W$ of the form

$$
W=\left[\begin{array}{ll}
0 & C \\
B & 0
\end{array}\right]
$$

where $B$ and $C$ are $m \times n$, and $n \times m$ submatricies, respectively. ( $m$ represents the number of alternatives and $n$ represents that of criteria.) Note that $W$ is irreducible because all the elements of $B$ and $C$ are positive. Finding the solution $\lambda$ of the equation

$$
S x=\lambda x
$$

where $\lambda$ is the maximum eigenvalue of $W$, we have the relative importance. If we regard the above equation as an eigenvalue problem, by the Perron-Frobenius theorem $x$ must be the unique positive solution except constant times. If the vector $x$ is separated to two subvectors $v$ and $w$, where $v$ corresponds to the relative importance of criteria and $w$ corresponds to that of alternatives, the equation (2) can be rewritten as

$$
\left[\begin{array}{ll}
0 & C \\
B & 0
\end{array}\right]\left[\begin{array}{l}
v \\
w
\end{array}\right]=\lambda\left[\begin{array}{l}
v \\
w
\end{array}\right]
$$

and hence we have

$$
B C w=\lambda^{2} w
$$

The next algorithm computes the relative importance $w$ : For given any nonnegative vector $u^{0} \neq 0$, the vector $u^{k}$ is generated by

$$
z^{k}=B C u^{k-1} \quad \text { and } \quad u^{k}=\frac{z^{k}}{\left\|z^{k}\right\|_{\infty}},
$$

where $\left\|z^{k}\right\|_{\infty}$ denotes the maximum norm of $z^{k}$. If we repeat the above procedure, $u^{k}$ will converge to a constant vector $u^{*}$ which corresponds to the relative importance $w$ of alternatives. The proof of convergence of this algorithm directly follows from the proof of Perron-Frobenius theorem. (Bertsekas and Tsitsiklis, 1989) 


\section{Procedure of revised ANP}

In this subsection, we summarize the procedure of revised ANP as follow:

(I) Ask a decision maker the aspiration level for each criterion.

(2) Consider the imaginary alternative which corresponds to the aspiration level.

(3) Perform pairwise comparisons under each criterion among alternatives consisting of actual alternatives and the imaginary alternative.

(4) Normalize the weights of alternatives by using the revised normalization.

(5) Construct a supermatrix without the weight of imaginary alternative.

(6) Computing the relative importance by the revised power method.

\section{Numerical Example}

In this section, we report the result of the revised ANP applied for small example. The example taken here is equivalent to AHP, because by removing the structural feature of ANP we are to show clearly that the revised ANP avoids the irrational rank reversal owing to the normalization procedure. For the comparison purpose, we also test the ordinary ANP for the same example. The results are shown in Tables $3 \sim 6$. In the Tables, $a_{l}(i=1,2,3)$ represent the alternatives and $c_{j}(j=1,2)$ represent the criteria. We assume that the weight of $c_{1}$ is 0.667 and that of $c_{2}$ is 0.333 .

Table 3 : Weights of alternatives

\begin{tabular}{|c|c|c|c|c|}
\hline & \multicolumn{2}{|c|}{ revised ANP } & \multicolumn{2}{c|}{ ordinary ANP } \\
\hline & $c_{1}$ & $c_{2}$ & $c_{1}$ & $c_{2}$ \\
\hline$a_{1}$ & 0.667 & 0.200 & 2 & $1 / 2$ \\
\hline$a_{2}$ & 0.333 & 0.800 & 1 & 2 \\
\hline
\end{tabular}

We first show in Table 3 that the result of pairwise comparison in the revised and ordinary ANP. Then the supermatricies $W_{1}$ in revised ANP and $W_{2}$ in ordinary ANP are generated as

$$
W_{1}=\left[\begin{array}{cccc}
0 & 0 & 0.667 & 0.667 \\
0 & 0 & 0.333 & 0.333 \\
2 & 1 / 2 & 0 & 0 \\
1 & 2 & 0 & 0
\end{array}\right], \quad W_{2}=\left[\begin{array}{cccc}
0 & 0 & 0.667 & 0.667 \\
0 & 0 & 0.333 & 0.333 \\
0.667 & 0.200 & 0 & 0 \\
0.333 & 0.800 & 0 & 0
\end{array}\right]
$$

respectively. From these supermatricies, we can obtain the relative importance of $a_{1}$ and $a_{2}$ which are shown in Table 4. Table 4 shows that $a_{1}$ is more important than $a_{2}$ in both ANP. The table also shows that the ratios of $a_{1}$ to $a_{2}$ are same in both ANP.

Table 4 : Relative importance

\begin{tabular}{|c|c|c|}
\hline & revised ANP & ordinary ANP \\
\hline$a_{1}$ & 1 & 0.541 \\
\hline$a_{2}$ & 0.888 & 0.489 \\
\hline
\end{tabular}


Next, we add an alternative $a_{3}$ and perform pairwise comparisons again. Table 5 shows that the result of pairwise comparịson in the revised and ordinary ANP.

Table 5 : Weights of alternatives

\begin{tabular}{|c|c|c|c|c|}
\hline \multirow{2}{*}{} & \multicolumn{2}{|c|}{ revised ANP } & \multicolumn{2}{c|}{ ordinary ANP } \\
\cline { 2 - 5 } & $c_{1}$ & $c_{2}$ & $c_{1}$ & $c_{2}$ \\
\hline$a_{1}$ & 2 & $1 / 2$ & 0.222 & 0.167 \\
\hline$a_{2}$ & 1 & 2 & 0.111 & 0.666 \\
\hline$a_{3}$ & 6 & $1 / 2$ & 0.667 & 0.167 \\
\hline
\end{tabular}

Then the supermatricies $W_{1}{ }^{\prime}$ in revised ANP and $W_{2}{ }^{\prime}$ in ordinary ANP are generated as

$$
W_{1}{ }^{\prime}=\left[\begin{array}{ccccc}
0 & 0 & 0.667 & 0.667 & 0.667 \\
0 & 0 & 0.333 & 0.333 & 0.333 \\
2 & 1 / 2 & 0 & 0 & 0 \\
1 & 2 & 0 & 0 & 0 \\
6 & 1 / 2 & 0 & 0 & 0
\end{array}\right], \quad W_{2}{ }^{\prime}=\left[\begin{array}{ccccc}
0 & 0 & 0.667 & 0.667 & 0.667 \\
0 & 0 & 0.333 & 0.333 & 0.333 \\
0.222 & 0.167 & 0 & 0 & 0 \\
0.111 & 0.666 & 0 & 0 & 0 \\
0.667 & 0.167 & 0 & 0 & 0
\end{array}\right],
$$

respectively. From these supermatricies, we can obtain the relative importance of $a_{1}, a_{2}$ and $a_{3}$ which are shown in Table 6. Table 6 shows that $a_{1}$ is more important than $a_{2}$ in revised ANP which $a_{2}$ is more important than $a_{1}$ in ordinary ANP. The table also shows that the ratio of $a_{1}$ and $a_{2}$ in revised ANP are the same as Table 4 but that in ordinary ANP' change significantly.

Table 6 : Relative imporfance

\begin{tabular}{|c|c|c|}
\hline & revised ANP & ordinary ANP \\
\hline$a_{1}$ & 0.360 & 0.204 \\
\hline$a_{2}$ & 0.320 & 0.296 \\
\hline$a_{3}$ & 1 & 0.500 \\
\hline
\end{tabular}

Therefore, it is verified that the revised ANP avoided the irrational rank reversal owing to normalization procedure in this example.

\section{Conclusion}

In this paper, we considered the irrational rank reversal of alternatives in ANP. One reason for such rank reversal is owing to the ordinary normalization procedure in which the weights sum to 1 . We first introduced the aspiration level which is the lowest level regarded by the decision maker, and then we proposed a revised normalization procedure using the aspiration level in which the weight of the aspiration level equals to 1 . We also proposed the revised power method to compute the relative importance since in the revised ANP the supermatrix may not be a stochastic matrix. Finally we showed that the irrational rank reversal owing to the normalization can be avoided in the proposed ANP. 


\section{References}

Belton, V. and Gear, T. (1983), "On a Short-coming of Saaty's Method of Analytic Hierarchies," OMEGA The International Journal of Management Science, 11, 3, 228-230.

Belton, V. and Gear, T. (1985), "The Legitimacy of Rank Reversal - a Comment," OMEGA The International Journal of Management Science, 13, 143-144.

Bertsekas, D. P. and Tsitsiklis, J. N. (1989) Parallel and Distributed Computation, Prentice-Hall, Englewood Cliffs, NJ.

Saaty, T. L. (1980) The Analytic Hierarchy Process, McGraw-Hill, NY.

Saaty, T. L. (1996) The Analytic Network Process, RWS publication, Pittsburgh. PA.

Tamura,H. Takahashi, S, Hatono, I and Umano, H. (1998), "On a Descriptive Analytic Hierarchy Process (D-AHP) for Modeling the Legitimacy of Rank Reversal (in Japanese), "Journal of the Operations Research Society of Japan, 41, 214-227. 